\title{
National trends in spinal arteriovenous malformations
}

\author{
A review
}

\author{
Shivanand P. Lad, M.D., Ph.D., ${ }^{1,2}$ Justin G. Santarelli, M.D., ${ }^{1,2}$ Chirag G. Patil, M.D., ${ }^{1,2}$ \\ Gary K. Steinberg, M.D., Ph.D., ${ }^{1,2}$ and Maxwell Boakye, M.D., \\ ${ }^{1}$ Department of Neurosurgery, Stanford University School of Medicine, Stanford; and ${ }^{2}$ Outcomes Research \\ Center, VA Palo Alto Health Care System, Palo Alto, California
}

\begin{abstract}
Object. Spinal arteriovenous malformations (AVMs) are rare and understudied vascular lesions that cause neurological insult by mass effect, venous obstruction, and vascular steal. These lesions are challenging entities to treat because of their complicated anatomy and physiology. Current management options include open microsurgery, endovascular embolization, and stereotactic radiosurgery.

Methods. Our study used the National Inpatient Sample database to analyze outcome data for spinal AVMs treated nationwide over an 11-year period from 1995 through 2006. Trends in procedural management, hospital course, and epidemiology of spinal AVMs are investigated.

Results. Annually, an average of 300 patients presented with spinal AVMs requiring hospital treatment. The average length of hospital stay for this treatment has declined from more than 9 days in 1995 to 6 days in 2006. However, the average cost of a hospital stay has increased from $<\$ 30,000$ to nearly $\$ 70,000$. Whereas one-half of spinal AVMs were treated operatively in 1995, one-third were managed operatively in 2006.

Conclusions. Spinal AVMs are being increasingly treated by endovascular, radiosurgical, or combined means. A discussion of modern strategies to treat these disorders is presented. (DOI: 10.3171/FOC.2009.26.1 E10)

\author{
KEY WORDS $\bullet \quad$ epidemiology $\bullet \quad$ length of stay $\bullet$
National Inpatient Sample $\bullet \cdot$ spinal arteriovenous malformation
}

$\mathrm{S}$ PINAL AVMs represent a unique category of pathological vascular lesions capable of manifesting severe neurological signs and symptoms. Typically these lesions cause pain, sensorimotor disturbance, or myelopathy by mass effect, hemorrhage, or vascular steal. Spinal AVMs are anatomically complex and often challenging to treat. Currently available therapeutic options include open microsurgery, endovascular embolization, and stereotactic radiosurgery. Management paradigms have changed over the years and are also institutionally dependent. Radiographically, the gold standard for spinal AVM diagnosis and characterization is catheter angiography; MR imaging and CT angiography are also diagnostically useful. $4,5,10,12$

Little has been written regarding the epidemiology of spinal AVMs. Our current study investigates the presentation and management of these vascular lesions nationwide from 1995 to 2006 . We report on and analyze the number of cases, type of treatment offered, length and cost of hospitalization, and patient age and sex at presen-

Abbreviations used in this paper: AVM $=$ arteriovenous malformation; LOS = length of stay; NIS = National Inpatient Sample. tation. Our findings add to the overall understanding of this rare and understudied entity.

\section{Methods}

We used the NIS database from the Healthcare Cost and Utilization Project, sponsored by the Agency for Healthcare Research and Quality, US Department of Health and Human Services. The NIS is a nationwide database of hospital inpatient stays representing the largest all-payer inpatient care database, which represents $\sim 20 \%$ of all inpatient admissions to nonfederal hospitals in the US. The NIS database contains data from $\sim 8$ million annual discharges from 1004 hospitals in 37 states. ${ }^{1}$ We analyzed data collected from 1995 through 2006 to determine general trends in hospitalizations and surgery for spinal AVMs across the US over this 11-year period.

The database was searched for hospital admissions categorized with a primary diagnostic code of 747.82 (spinal vascular malformation) according to the International Classification of Diseases, Ninth Revision, Clinical Modification (ICD-9-CM). Outcome measures included total discharges, LOS, total hospital charges, and the 


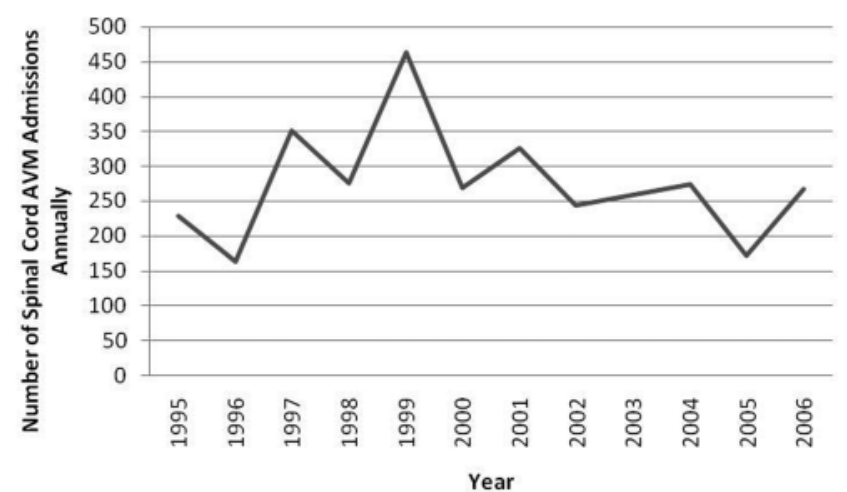

FIG. 1. Line graph depicting the number of hospital admissions for spinal AVMs from 1995 to 2006.

"national bill" (total discharges $\times$ total hospital charges). We also examined patient demographics such as age, sex, race, and median household income for zip code, as well as primary payer (Medicare, Medicaid, or private insurance), type of admission, and hospital region, size, and teaching status, all of which were coded in the NIS data. Statistically significant differences between variable means and their corresponding standard errors were evaluated using the Z-test. A probability value $<0.05$ was considered statistically significant. All data analyses were performed using SAS version 9.1 (SAS Institute, Inc.).

\section{Results}

A total of 3291 patients were admitted with the diagnosis of spinal vascular malformation over the 11-year period of our study. Temporal trends in the volume of admissions for patients with spinal AVMs during this interval are illustrated in Fig. 1.

On average, 299 patients were annually admitted to hospitals nationwide with the new diagnosis of spinal AVM. Admissions reached a peak in 1999, when $>450$ such patients presented to medical attention, and a nadir in 1995 and 2005, with < 200 patients each year. In 2006, there were 267 spinal AVM admissions reported.

Length of hospital stay following spinal AVM admission and management was also reported. In the first year of this study, average LOS was noted to be 9.5 days, lon-

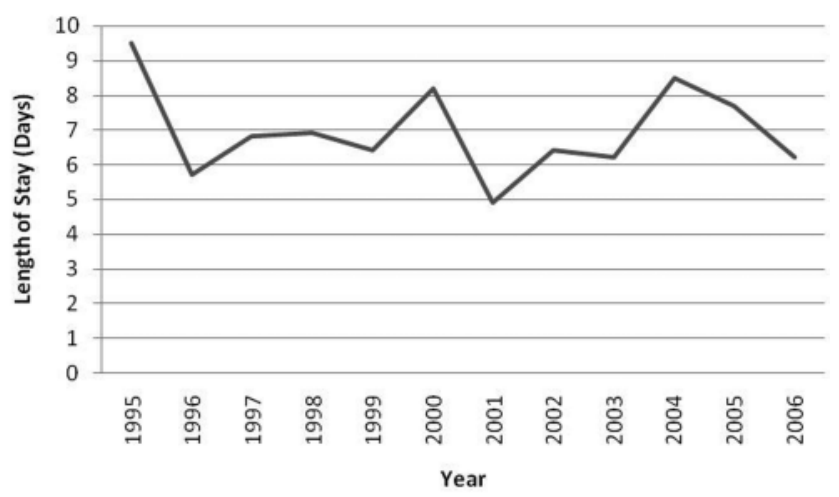

FIG. 2. Line graph showing the average LOS for hospitalized patients with spinal AVMs from 1995 to 2006.

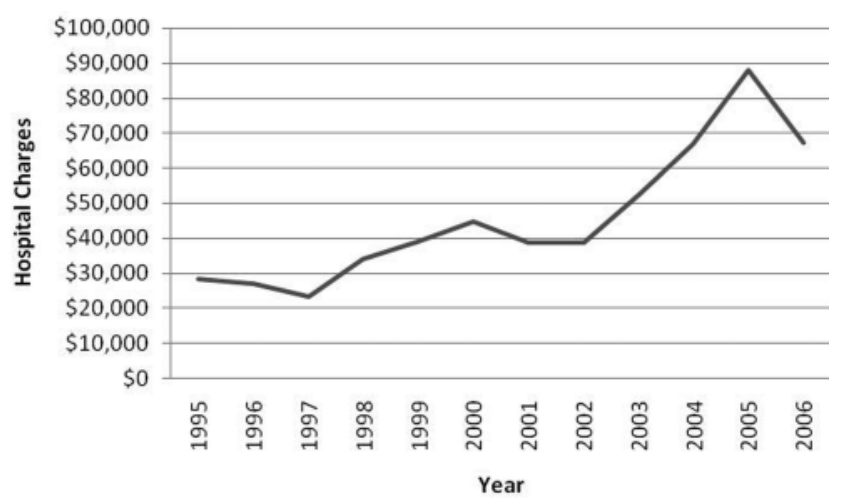

FIG. 3. Line graph depicting the average cost of a hospital stay for hospitalized patients with spinal AVMs from 1995 to 2006.

ger than any subsequent year. By 2006, LOS had fallen to 6.2 days (Fig. 2).

Over the 11-year time span, the average cost of admission and treatment increased dramatically. In 1995, total hospital charges amounted to $\$ 28,308$ on average. By 2006, costs had reached \$67,102 (Fig. 3).

Table 1 depicts categorical specifics for all spinal AVM admissions in 2006, the most recent year studied. Of the 267 total admissions, the most common age group represented was the 45 - to 64-year-old population (36\%), followed by 18 - to 44 -year-olds (24\%), 1 - to 18 -year-olds (22\%), and those 65 years of age and older (18\%; Fig. 4). Hospital charges did not vary significantly among age groups, although LOS was notably longest in the eldest age category (6.9 vs 3.9 days for other groups). There were more male patients $(57 \%)$ than female patients $(43 \%)$. According to the insurance coverage analysis, Medicare accounted for $40 \%$ of all payers, whereas private insurance covered nearly $50 \%$ of claims (Table 1). The median income of most patients was $>\$ 36,000$. In 2006, the vast majority of patients with spinal AVMs were treated at academic/teaching medical centers (81\%), classified as large hospitals (78\%) in metropolitan areas (98\%) in the US (Table 1).

Choice of management of spinal AVMs changed $\sim 49 \%$ of spinal AVM admissions were treated surgically.

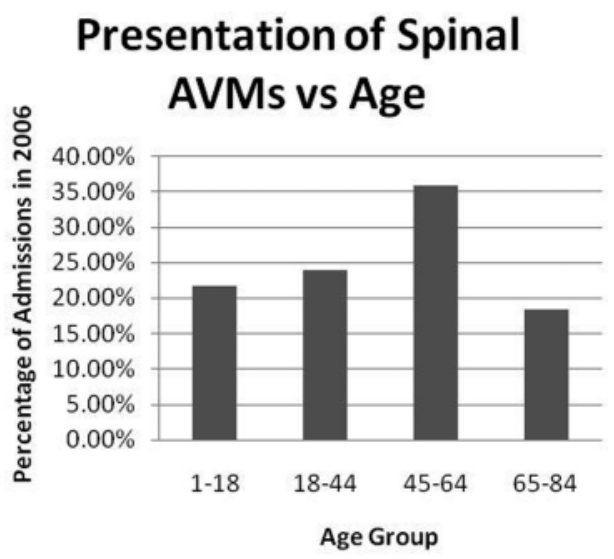

FIG. 4. Bar graph showing the stratification of ages among all patients admitted to hospitals for management of spinal AVMs 
A review of national trends in spinal AVMs

TABLE 1: Patient demographics for spinal cord AVM admissions in 2006*

\begin{tabular}{|c|c|c|c|c|c|}
\hline Variable & $\begin{array}{c}\text { Total No. of } \\
\text { Discharges (\%) }\end{array}$ & $\begin{array}{l}\text { Mean LOS } \\
\text { (days) }\end{array}$ & $\begin{array}{c}\text { Mean } \\
\text { Charges (\$) }\end{array}$ & "National Bill” (\$) & $\begin{array}{c}\text { Routine } \\
\text { Discharge \% }\end{array}$ \\
\hline all discharges & $267(100)$ & 6.2 & 67,102 & $17,916,234$ & 61.46 \\
\hline \multicolumn{6}{|l|}{ age group } \\
\hline $1-18$ & $58(21.72)$ & NA & 62,592 & $3,630,336$ & NA \\
\hline $18-44$ & $64(23.97)$ & 3.9 & 55,445 & $3,548,480$ & NA \\
\hline $45-64$ & $96(35.96)$ & 3.9 & 62,023 & $5,954,208$ & NA \\
\hline $65-84$ & $49(18.35)$ & 6.9 & 71,975 & $3,526,775$ & NA \\
\hline \multicolumn{6}{|l|}{ sex } \\
\hline male & $153(57.30)$ & 4.4 & 61,202 & $9,363,906$ & 56.51 \\
\hline female & $114(42.70)$ & NA & 75,200 & $8,572,800$ & 68.13 \\
\hline \multicolumn{6}{|l|}{ insurance payer } \\
\hline medicare & $107(40.07)$ & 6.6 & 66,981 & $7,166,967$ & NA \\
\hline private insurance & $130(48.69)$ & 4.4 & 66,737 & $8,675,810$ & 72.41 \\
\hline \multicolumn{6}{|l|}{ patient income } \\
\hline not low $(\geq \$ 36,000)$ & $221(82.77)$ & 6.7 & 71,995 & $15,910,895$ & 61.82 \\
\hline \multicolumn{6}{|l|}{ hospital owner } \\
\hline private, not-for-profit & $222(83.15)$ & 6.7 & 71,915 & $15,965,130$ & 61.80 \\
\hline \multicolumn{6}{|l|}{ hospital teaching status } \\
\hline teaching & $216(80.90)$ & 6.7 & 75,726 & $16,356,816$ & 65.23 \\
\hline \multicolumn{6}{|l|}{ hospital location } \\
\hline metropolitan & $262(98.13)$ & 6.2 & 65,985 & $17,288,070$ & 62.67 \\
\hline \multicolumn{6}{|l|}{ hospital size (beds) } \\
\hline large & $208(77.90)$ & 5.6 & 69,127 & $14,378,416$ & 58.07 \\
\hline
\end{tabular}

*"National Bill" = total discharges $\times$ total hospital charges. Abbreviation: NA = complete data not available.

By 2006, only one-third of admissions (33\%) were operatively managed (89 of 267 patients; Fig. 5).

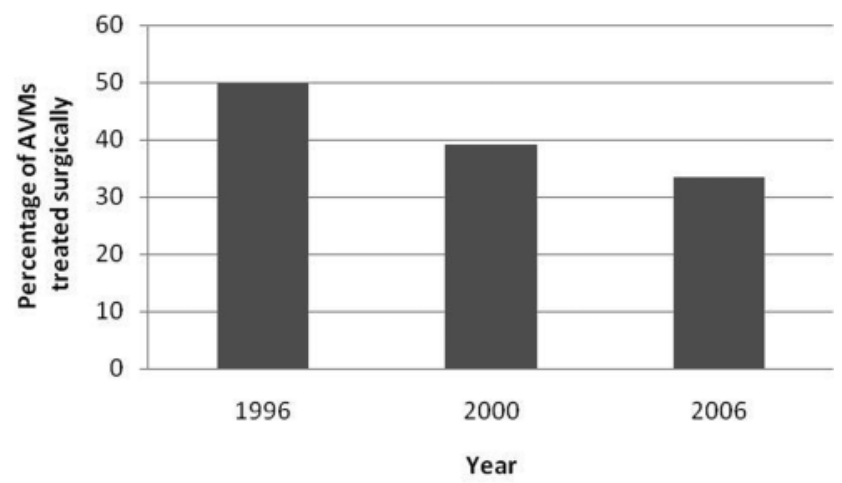

FIG. 5. Bar graph showing the decline in the percentage of patients with spinal AVMs treated by surgery alone.

\section{Discussion}

Spinal AVMs are vascular lesions capable of inflicting serious neurological sequelae secondary to mass effect, hemorrhage, or vascular steal phenomenon. ${ }^{2,7}$ Our present study analyzed trends in the management of spinal AVMs over the 11-year span from 1995 to 2006. This interval of time saw advances in endovascular techniques and in the use of stereotactic radiosurgery to complement or supercede open surgery. Trends are reported not only for the type of treatment selected, but also for LOS and total hospital charges, as well as for age and sex classification, insurance status, and type of hospital in which treatment occurred. One notable finding of this study included the dramatic increase in hospital costs over the 11 -year time frame, from $<\$ 30,000$ per patient in 1995 to nearly $\$ 70,000$ in 2006 , despite an overall reduction in LOS from $>9$ days to $<7$ days per patient.

Debate exists over the best method of classifying spinal AVMs. The longest established classification categorizes these lesions into 4 broad types. Type 1 refers to 
dural arteriovenous fistulas (which, in fact, are not true AVMs). Type 1 lesions most commonly are identified in the lower thoracic cord or the conus medullaris and consist of a single arterial feeder vessel that drains into a dural vein..$^{14}$ They may be spontaneous or traumatic in nature. ${ }^{14}$ Type 2 spinal AVMs are also called "glomus malformations" and are intramedullary in location; they are most frequently seen at the cervicomedullary junction. ${ }^{18}$ Type 3 spinal AVMs are referred to as juvenile or metameric. They are often vast in dimension, span multiple vertebral levels, and may extend significantly into the extraspinal space. ${ }^{9}$ Finally, Type 4 lesions are perimedullary, and are typically ventral (supplied by the anterior spinal artery). ${ }^{6}$

Whereas surgical management of spinal AVMs was undertaken in approximately one-half of the reported cases in 1995, this rate fell to one-third of cases by 2006. It is unclear how many patients on a national scale were treated by radiosurgery or embolization, or by a combination of available techniques. Nonetheless, a trend toward less invasive management is noticeable. Interdisciplinary team management by neurosurgeons and neuroradiologists is essential to evaluate patients for surgical, endovascular, or combined treatment on a case-by-case basis. ${ }^{3,6,9,11,15}$ Consensus still appears to favor open microsurgery for many Type 1 spinal AVMs. For other variants of Type 1 spinal AVMs, however, embolization may be an appropriate option, ${ }^{11,17}$ particularly when the dural artery originates from a branch supplying the spinal cord. Spinal AVM Types 2 and 4 appear better suited in general to endovascular embolization. Type 3 spinal AVMs are anatomically complicated, and not readily surgically or endovascularly managed. Several authors have reported on a combined open surgical and endovascular approach for treatment of these so-called Type 3 metameric lesions. ${ }^{8,13,16}$

Endovascular management of spinal AVMs has evolved due to the development of a new generation of microcatheters and embolic materials. ${ }^{17}$ Liquid embolic agents have become a first choice for embolization. The adhesive $N$-butyl cyanoacrylate is a preferred embolic agent, although the liquid embolic agent Onyx has been used with success as well, including in the management of difficult intramedullary spinal AVMs in 2 or fewer treatment sessions. ${ }^{3}$ Radiotherapy has also gained credence in the treatment of spinal AVMs. Several authors have reported success at treating these lesions over a long period of time..$^{8,15}$

\section{Conclusions}

The NIS database provides a valuable opportunity for examining trends in the frequency of procedures and population characteristics for a number of different diagnoses. We studied a representative sample of patients who were admitted and underwent surgery for spinal AVMs between 1995 and 2006 in the US. The NIS database has several unique advantages as well as some shortcomings. The main advantage of the NIS database is its comprehensive sampling of the entire US nonfederal hospital community. Most of the previously published literature on spinal vascular malformations reflects the experience of single institutional studies. However, the NIS database avoids selection bias on both an individual and institutional level. This type of administrative database also has some inherent limitations. Only inpatient data are available during each hospitalization, and frequency of embolization or combination embolization/surgery is difficult to determine due to limitations in coding. Because individual patients cannot be identified or followed after discharge, outcome measures other than discharge status (such as long-term functional outcome) are not available. In addition, detailed clinical information (such as duration and severity of symptoms before surgery) is not available. Nonetheless, this database provides us with useful information regarding trends in the management of spinal AVMs on a national level and provides fodder for discussion of the pathology, natural history, and changing management paradigm of these understudied lesions. Whereas open surgery will remain the mainstay treatment for certain types of spinal AVMs, endovascular embolization and stereotactic radiosurgery have become increasingly validated on a national and international scale.

\section{Disclosure}

The authors report no conflict of interest concerning the materials or methods used in this study or the findings specified in this paper.

\section{References}

1. Agency for Healthcare Research and Quality: Calculating Nationwide Inpatient Sample Variances. Rockville, MD: Agency for Healthcare Research and Quality, 2002 (http:// www.hcup-us.ahrq.gov/db/nation/nis/reports/NIS_2000_ Design_Report.pdf ) [Accessed 2 January 2009]

2. Aminoff MJ, Barnard RO, Logue V: The pathophysiology of spinal vascular malformations. J Neurol Sci 23:255-263, 1974

3. Corkill RA, Mitsos AP, Molyneux AJ: Embolization of spinal intramedullary arteriovenous malformations using the liquid embolic agent, Onyx: a single-center experience in a series of 17 patients. J Neurosurg Spine 7:478-485, 2007

4. Detweiler PW, Porter RW, Spetzler RF: Spinal arteriovenous malformations. Neurosurg Clin N Am 10:89-100, 1999

5. Ferch RD, Morgan MK, Sears WR: Spinal arteriovenous malformations: a review with case illustrations. J Clin Neurosci 8:299-304, 2001

6. Heros RC, Debrun GM, Ojemann RG, Lasjaunias PL, Naessens PJ: Direct spinal arteriovenous fistula: a new type of spinal AVM. Case report. J Neurosurg 64:134-139, 1986

7. Kataoka H, Miyamoto S, Nagata I, et al: Venous congestion is a major cause of neurological deterioration in spinal arteriovenous malformations. Neurosurgery 48:1224-1230, 2001

8. Kim LJ, Spetzler RF: Classification and surgical management of spinal arteriovenous lesions: arteriovenous fistulae and arteriovenous malformations. Neurosurgery 59 (5 Suppl): S195-S201, 2006

9. Krings T, Mull M, Gilsbach JM, et al: Spinal vascular malformations. Eur Radiol 15:267-278, 2005

10. Marsh WR: Vascular lesions of the spinal cord: history and classification. Neurosurg Clin N Am 10:1-8, 1999

11. Niimi Y, Berenstein A: Endovascular treatment of spinal vascular malformations. Neurosurg Clin N Am 10:47-71, 1999

12. Oldfield EH, Doppman JL: Spinal arteriovenous malformations. Clin Neurosurg 34:161-183, 1988

13. Rodesch G, Lasjaunias P: Spinal cord arteriovenous shunts: 
A review of national trends in spinal AVMs

from imaging to management. Eur J Radiol 46:221-232, 2003

14. Rosenblum B, Oldfield EH, Doppman JL, Di Chiro G: Spinal arteriovenous malformations: a comparison of dural arteriovenous fistulas and intradural AVMs in 81 patients. J Neurosurg 67:795-802, 1987

15. Sinclair J, Chang SD, Gibbs IC, Alder JR Jr: Multisession CyberKnife radiosurgery for intramedullary spinal cord arteriovenous malformations. Neurosurgery 58:1081-1089, 2006

16. Spetzler RF, Detwiler PW, Riina HA, Porter RW: Modified classification of spinal cord vascular lesions. J Neurosurg 96:145-156, 2002

17. Veznedaroglu E, Nelson PK, Jabbour PM, Rosenwasser RH:
Endovascular treatment of spinal cord arteriovenous malformations. Neurosurgery 59 (5 Suppl):S202-S209, 2006

18. Zozulya YP, Slin'ko EI, Al Q II: Spinal arteriovenous malformations: new classification and surgical treatment. Neurosurg Focus 20(5):E7, 2006

Manuscript submitted September 15, 2008

Accepted October 28, 2008.

Address correspondence to: Shivanand P. Lad, M.D., Ph.D., Department of Neurosurgery, Stanford University Medical Center/ VA Palo Alto Health Care System, 3801 Miranda Avenue, M-112, Palo Alto, California 94304. email: nlad@stanford.edu. 